\title{
Ética e historia: una mirada desde la ética discursiva
}

\section{Ethics and history: A look from the discursive ethics Ética e história: uma visão de ética do discurso}

\author{
Dr. Conzalo Scivoletto ${ }^{1}$
}

Recibido: 23/01/2017 - Aceptado: 28/02/2017

\begin{abstract}
Resumen
El siguiente trabajo se propone analizar la relación entre la ética discursiva y la historia, a partir de la reconstrucción realizada por Karl-Otto Apel en las Mercier Lectures de 1999 (Lovaina). Se intentará mostrar cómo la ética discursiva se encuentra entrelazada con las tres dimensiones del tiempo histórico. La relación con el pasado apunta al posicionamiento respecto de la situación moral e institucional "heredada" por la tradición (eticidad sustancial). La relación con el presente se refiere a la "situación humana", entendida esta como un proceso evolutivo-cultural (desde la hominización hasta el presente) y como un "momento" o situación particular dentro de ese proceso. Finalmente, la relación con el futuro alude a la capacidad de la ética de orientar la historia humana hacia un horizonte normativo, en tanto ideal regulativo.
\end{abstract}

Palabras clave: antropología - institucionalización - filosofía práctica

\begin{abstract}
This work aims to analyze the relationship between discursive ethics and history, based on the reconstruction by Karl-Otto Apel in the 1999 in Mercier Lectures (Leuven). It attempts to show how discursive ethics is intertwined with the three dimensions of historical time. The relationship with the past points to the positioning regarding the moral and institutional situation "inherited" by tradition (substantial ethics). The relationship with the present refers to the "human situation", understood
\end{abstract}

1 Argentino, Dr. en Filosofía por la Universidad Nacional de Lanús. Becario postdoctoral de Conicet con lugar de trabajo en el Instituto de Ciencias Humanas Sociales y Ambientales (INCIHUSA) perteneciente al Consejo Nacional de Investigaciones Científicas y Técnicas (CONICET). Contacto: scivolettog@gmail.com 
as an evolutionary-cultural process (from hominization to the present) and as a "moment" or particular situation within that process. Finally, the relationship with the future alludes to the ability of ethics to guide human history towards a normative horizon, as a regulative ideal.

Keywords: Anthropology - institutionalization - practical philosophy

\section{Resumo}

O seguinte artigo propõe analisar a relação entre a ética do discurso e história, desde a reconstrução realizada por Karl-Otto Apel nas Mercier Lectures de 1999 (Lovaina). Ele tentará mostrar como a ética do discurso se encontra entrelaçada com as três dimensões do tempo histórico. A relação com o passado aponta ao posicionamento sobre a situação moral e institucional "herdada" pela tradição (ética substancial). A ligação com o presente refere-se à "situação humana", entendida como um processo evolutivo-cultural (a partir da hominização presente) e como um "momento" ou situação particular dentro daquele processo. Finalmente, a relação com o futuro se refere à capacidade da ética para orientar a história humana para um horizonte normativo, enquanto ideal regulador.

Palavras-chave: antropologia - institucionalização - filosofia prática

\section{Introducción}

Desde la primera exposición de la ética del discurso en 1973, entonces ética de la comunicación (1985 II 341-413), Apel se ha encargado de mostrar no solo los argumentos filosóficos que la fundamentan, sino también cómo se relaciona con el contexto histórico-social (Apel 19982001 2007). En sus comienzos, es decir, a fines de los años 60, generalmente se entendía por contexto los desafíos de una sociedad técnica global que ponía en peligro la supervivencia de la humanidad misma. En ello, sin duda, resonaban tanto Heidegger como los pensadores de la escuela de Frankfurt. Con el paso del tiempo, esa crítica hacia la sociedad tecno-científica se fue ampliando hacia otros asuntos. Uno en particular merece ser destacado: el conflicto Norte-Sur, en el cual Apel es interiorizado gracias a sus debates con Enrique Dussel (Apel-Dussel, 2005). Ahora, más allá de cuáles sean empíricamente los grandes problemas que han ido aquejando a la humanidad desde mediados del siglo XX, el punto es que Apel entiende a la ética no como una actividad filosófica "pura", que evoluciona por resolución 
de problemas inmanentes a la disciplina, sino que la piensa más bien como una "respuesta" de la razón a los desafíos "externos" con los que se enfrenta, sean estos la crisis nuclear, la pobreza y la desigualdad o la contaminación del planeta. El punto de partida o base para tal consideración, Apel la encuentra en la antropología y la teoría de la evolución proveniente, sobre todo, de la antropología alemana clásica (von Uexküll, Scheler, Gehlen, etc.). ${ }^{2}$ En las Mercier Lectures ${ }^{3}$, texto en el que me centraré principalmente en este trabajo, Apel estructura su introducción a la ética del discurso en función de esta hipótesis general: la razón práctica o la ética es una respuesta a los desafíos con los que se enfrenta el hombre dada su condición o situación antropológica. La historia de la ética, entonces, incluida las religiones como fuente de normatividad, se relaciona dialécticamente, por así decir, con dicha situación.

Desde mi punto de vista, las ocho conferencias que componen el texto publicado de las Mercier Lectures pueden ser divididas en tres grandes bloques. El primero correspondería a las primeras dos conferencias, las cuales se ocupan del problema antropológico en general: aquí Apel discute con von Uexküll, Gehlen y sociobiólogos más recientes como Dawkins sobre el origen y función de la moralidad en la cultura. En el segundo bloque, desde la tercera a la sexta conferencia, Apel presenta cómo se realiza concretamente la dialéctica entre "desafíos externos" $y$ "recursos internos" de la razón en dos momentos particularmente acuciantes del siglo XX: el primero, en torno a 1945, y el segundo, de mediados de 1970. Finalmente, el tercer bloque, que corresponde a las últimas dos conferencias, apunta a mostrar cómo la ética del

2 Más allá de los títulos que se mencionan en este trabajo, es difícil encontrar en Apel una antropología propiamente dicha. Con lo que nos podemos encontrar es, más bien, con algunas afirmaciones o presupuestos antropológicos que es posible reconstruir hasta cierto punto, pero sobre todo es necesario continuar. Un punto de partida posible para una antropología discursiva se encuentra en: Burckhart, 1999; Niquet, 2002. También Maliandi, a mi juicio, desarrolla una antropología convergente con la de Apel; ver Maliandi 1984.

3 Estas conferencias o lecciones fueron impartidas por Apel en inglés en marzo de 1999 en el marco de la Chaire Cardinal Mercier del Institut supérieur de philosophie de la Universidad Católica de Lovaina. 
discurso (fundamentada en las conferencias anteriores, y presentada como una respuesta pertinente a los desafíos externos y superadora de los recursos internos) puede intervenir en la historia a través de su aplicación. Ciertamente, la importancia de estas conferencias no radica en su originalidad o abordaje temático, puesto que aquí Apel reúne reflexiones e incluso fragmentos de sus propios textos en orden a una exposición de conjunto de la ética del discurso. Pero es precisamente ese carácter de "visión de conjunto" y de introducción el que resulta relevante, en tanto forma de presentación o exposición sistemática. Pero, además, también es importante destacar que se trata de la última, cronológicamente hablando, exposición de conjunto de la ética del discurso realizada por Apel.

Sin embargo, en este trabajo no me ocuparé de la sistematización o arquitectónica de la ética del discurso, tampoco de su fundamentación ni, por así decir, de su evolución interna, sino que me interesa analizar más bien una cuestión muy puntual, a saber, cómo se relaciona con la historia. Lo que intentaré aclarar es qué puede significar concebir la ética del discurso como una ética de la corresponsabilidad relativa a la historia. Siguiendo a Apel, y particularmente las Mercier Lectures ya mencionadas, creo que ese "relativo a" puede significar dos cosas: referida a la historia de la humanidad, de la cultura y la sociedad en general; $y$ referida a la historia de la filosofía y de la ética en particular, como forma de respuesta a los desafíos planteados por esa historia de la cultura o de la sociedad. Ahora bien, intentaré mostrar, además, que esta doble relación dialéctica de la ética con la historia puede ser divida en los tres momentos de la historicidad o "éxtasis temporales". Así, la ética se ocuparía de realizar un diagnóstico del presente, una crítica de la tradición y de las instituciones y de aportar criterios para el progreso moral. La tarea histórica que desarrolla la ética del discurso (que no es otra cosa que su aplicabilidad) se da, entonces, simultáneamente en las tres dimensiones del tiempo: pasado, presente y futuro. ${ }^{4}$

4 A ello se podría objetar que toda ética filosófica desempeña una tarea histórica, también en las tres dimensiones del tiempo. Sin embargo, creo que esto no es así. Por ejemplo, la ética kantiana (como ya vio Hegel) se desconecta del "pasado" en el 


\section{La ética y la situación humana}

La relación de la ética o de la racionalidad práctica con la "situación humana" es un tema que Apel ha abordado en otros lugares, fundamentalmente en "Die Situation des Menschen als ethisches Problem" (Apel 1988 42-68). Por situación humana se entiende, en primer lugar, aquello que concierne a la humanidad "como un todo". En la conferencia mencionada Apel se refiere al peligro de la guerra nuclear y la crisis ecológica. En ambos casos se trata, como claramente se puede ver, de situaciones que ponen en riesgo a la humanidad y que demandan de esa humanidad una respuesta global. La "globalidad" o universalidad de la afectación entonces puede ser considerada como un rasgo distintivo: un macro problema requiere de una macro ética. Es decir que "situación humana" en este primer sentido es una realidad actual, un diagnóstico del presente según el cual la supervivencia ${ }^{5}$ de todos está en juego. Pero hay otro sentido, al que podríamos denominar estructural, y que se fundamenta en una condición antropológica, pero que al mismo tiempo no es a-histórica, sino que se trata de un proceso evolutivo-cultural. En las Mercier Lectures, Apel reconstruye

sentido de la eticidad heredada y encarnada en las instituciones. La ética aristotélica, y de ahí la hermenéutica, pone el acento en esas tradiciones o el ethos moral de una comunidad, sin embargo, no cobra la misma relevancia la dimensión "utópica" del futuro. Si bien estas consideraciones tan genéricas ameritan una justificación detallada, que por supuesto no puedo desarrollar aquí por exceder ampliamente los límites de este trabajo, sí me parece importante destacar, al menos, el criterio de la relación con el pasado, el presente y el futuro como una forma de establecer una comparación que no sea meramente formal entre las teorías éticas. En efecto, sobre todo para quienes nos interesa pensar la teoría ética desde su aplicabilidad (¿y si no para qué la ética?), este criterio nos permitiría tener una visión más amplia y más profunda de su alcance práctico y posibilidades de transformación de la realidad. Los dilemas a los que se habría de enfrentar la ética, en cuanto a su aplicabilidad, serían, de acuerdo con este criterio: dependencia/independencia del juicio moral (respecto del pasado), singularidad/no singularidad (respecto de la situación presente), posibilidad/imposibilidad del progreso (en sentido práctico o normativo).

5 Este es, según Apel, uno de los dos principios regulativos de la acción humana o de la "estrategia moral" a largo plazo: garantizar la supervivencia de la comunidad real de comunicación. El otro principio consiste en la realización de la comunidad ideal en la real, con lo cual, la estrategia de emancipación le otorga sentido a la estrategia de supervivencia. (Apel 1985 II 408ss). 
esta perspectiva estructural (antropológica) en cuatro pasos o cursos ${ }^{6}$. En el primero se ocupa de la hominización, y de cómo con el pasaje del instinto a la cultura se presenta por primera vez un desafío a la razón para dar respuesta a los desafíos que tal pasaje conlleva. Como muestra Apel, ya Kant y Herder, entre otros, se habían preguntado por el origen de la moral y por este límite o transición del instinto a la moralidad. Pero es sobre todo con la antropología filosófica del siglo XX cuando este problema asume un carácter sistemático.

Dentro de esa gran tradición, uno de los principales exponentes con quien discute Apel es Arnold Gehlen7. De acuerdo con Gehlen, y a diferencia de Kant, no es la "razón" quien reemplaza la conducta instintiva animal sino las instituciones. De hecho, las instituciones liberarían al hombre de la "carga" de la toma de decisiones mediante la estabilización de reglas de conducta que son, por así decir, independientes de la voluntad subjetiva. Apel, si bien considera atendible esta interpretación antropológica del origen de la moral, no acuerda con el carácter puramente institucionalista, y por lo tanto conservador, de Gehlen. Para Apel, también desde el comienzo de la historia la crítica de las instituciones ha conformado más radicalmente lo que podríamos llamar la "razón práctica". Desde un punto de vista histórico, los diferentes procesos de llustración, por ejemplo, en la época axial (Jaspers) de Grecia, China o India, la filosofía (en sentido amplio) se encargó de cuestionar las tradiciones y las instituciones de base religiosa, esto es, dogmática.

El segundo momento de reconstrucción se refiere a la "carrera del homo faber" hasta el presente (Apel 2001 8-12). El homo faber sería

\footnotetext{
6 Aquí me ocuparé sobre todo de los dos primeros momentos, que se corresponden con los desafíos "externos" desde un punto de vista antropológico. Los pasos o momentos tercero y cuarto se ocupan de los "recursos internos" con los que cuenta la razón para enfrentarse a los desafíos externos. En relación con la "hominización" y la aparición de la técnica, Apel aquí menciona a la ilustración griega y lo que Jaspers Ilama la época axial, interpretado por Apel como un intento de ética o racionalidad práctica post-convencional, especialmente destacada en China, India y Grecia.

7 Para la interpretación crítica de Apel de la antropología de Gehlen, ver Apel 1985 I 191-214.
} 
un "punto límite" en la medida que transgrede las barreras del instinto e interfiere en el mundo natural a través de herramientas, de allí su posibilidad de transformar completamente su medio, de medir, prever, calcular beneficios y costos. La racionalidad, en este sentido, se caracteriza por la manipulación y el dominio del mundo estableciendo relaciones medio-fin. Por lo tanto, la aparición de la técnica sería una respuesta, un mecanismo de adaptación de la especie humana a un medio adverso; es decir, frente a la posibilidad de no supervivencia y de recursos biológicos escasos (pelaje, colmillos, velocidad, etc.) habríamos desarrollado otros recursos artificiales, como la vestimenta, el cuchillo, las canoas, etc. Pero, paradójicamente, con el consecuente avance de la técnica se acrecienta, ya desde el comienzo de la civilización, la capacidad de destrucción tanto del medio ambiente como de los propios semejantes; por ejemplo, el hacha que le habría permitido al hombre primitivo construir embarcaciones, al mismo tiempo, pudo convertirse en un arma letal para los otros seres humanos. De acuerdo con ello, y siempre a partir de este enfoque antropológico, el medio de compensación por las consecuencias de la utilización de herramientas y de su peligrosidad para la propia especie fueron en un comienzo los grandes sistemas religiosos y filosóficos del mundo antiguo, que pudieron dar, a su modo, un fundamento a la invención de normas o principios de acción. Para Apel, es la propia racionalidad técnica del homo faber la que "impulsa" la necesidad de "ampliación" de esa racionalidad. En conclusión, la ética (racionalidad práctica) surgiría como una respuesta del hombre a su propia situación o su posición en el mundo. El diagnóstico del presente, el hecho de encontrarnos sumidos bajo el imperio de la técnica que pone en peligro la humanidad entera, se encuentra proyectado por esta estructura antropológica. "Nuestra situación", ahora sí, histórica y particular (desde la segunda mitad del siglo XX), es una agudización de la situación humana como tal. Al comienzo de la tercera conferencia en las Mercier Lectures, Apel afirma:

Quiero mostrar que, por un lado, los desafíos externos a la ética, los cuales se incrementaron y agudizaron a lo largo de la evolución cultural, en el siglo XX alcanzaron un punto máximo no experimentado hasta entonces, y que, por otro lado, los recursos internos de la ética filosófica no fueron de 
ninguna manera capaces de lidiar con los nuevos desafíos de la situación [...] (Apel 2001 27).

Como se puede observar, la condición antropológica estructural no es ajena a la particularidad de nuestro momento histórico, es por eso que cuando Apel habla de "situación humana", lo hace, según entiendo, en estos dos sentidos conectados: como condición antropológica y como un momento, dentro de un proceso evolutivo, de dicha condición. El hombre "necesita" de la moral también en esos dos sentidos: como una respuesta antropológicamente necesaria y como una respuesta epocal-particular en la que se plasma tal condición. Pero lo peculiar de nuestra situación hoy es que ya no es suficiente con la moralidad institucional convencional. ${ }^{8}$ Dado el carácter global de la crisis presente y la ruptura con un orden institucional cerrado y, por lo tanto, la apertura de un mundo pluralmente cosmovisivo, la respuesta ética debe asumir también un carácter global que aspire a una fundamentación posconvencional. ${ }^{9}$ Sin embargo, posconvencional no quiere decir desinstitucionalizado: la moral debe también institucionalizarse para que tenga sentido hablar de una transformación social que alcance el doble objetivo de la supervivencia (de una comunidad real de comunicación) y de la emancipación (la realización de una comunidad ideal de comunicación). Es por eso que, a mi juicio, la ética discursiva no es una ética "personalista", no pone el acento primordialmente en las condiciones subjetivas de los agentes morales, sino en las condiciones marco para que agentes morales (esto es, agentes que son imputables) puedan desempeñarse como tales.

Apel, como adelanté, analiza dos momentos particulares que representan nuestra situación humana "hoy" en tanto agudización de la situación humana como tal: la situación derivada inmediatamente

\footnotetext{
8 Para la interpretación apeliana de la lógica del juicio moral, que parte de Piaget y Kohlberg, ver, sobre todo, Apel 1988 306-369.

9 Dentro del esquema de Kohlberg, la moral convencional se refiere a las expectativas de grupos y la lealtad a una comunidad como garantía del orden social. En el nivel posconvencional, el juicio moral se desarrolla con autonomía y a partir de principios en sí mismo universales y que exceden la moral establecida de un grupo o comunidad política (incluso un Estado).
} 
de la Segunda Guerra Mundial y la crisis económico-ecológica desde fines de la guerra fría. En ambos momentos se manifiestan crisis de alta relevancia para la humanidad entera y en ambos casos las filosofías "vigentes" no pueden dar cuenta o dar una respuesta acabada a tales desafíos.

\section{La ética y las instituciones}

En el punto anterior he intentado mostrar, de acuerdo con Apel, cómo se vincula la ética discursiva con el presente, cómo tal vinculación consiste en una relación con la situación humana actual y que esa situación actual forma parte de la situación humana como tal, en tanto proceso antropológico-evolutivo. Pero ya en este análisis se planteó que la función propia de la razón práctica o de la filosofía es la de una crítica a las instituciones. En este sentido, la relación con el pasado, con la eticidad "heredada" es la de la distancia crítica. Pero también la ética discursiva entabla una particular relación con la tradición filosófica, en tanto recurso interno para responder a los desafíos externos y ejercer esa crítica de la tradición heredada. A continuación, quisiera entonces referirme a esos dos puntos: la relación de la ética con las tradiciones y las instituciones y la relación de la propia ética discursiva con la tradición filosófica (ética).

Tal como había anticipado, Apel, en el segundo bloque de las Mercier Lectures se ocupa del contexto (como desafío externo) de la posguerra y el mundo bipolar y de la globalización económica desde 1970 hasta el presente. Ambos contextos fueron abordados por la filosofía (como recurso interno): en el primer caso, por el racionalismo científico y el existencialismo, como filosofías dominantes en el occidente capitalista, y el materialismo dialéctico como filosofía representativa del bloque comunista. ${ }^{10}$ En el segundo periodo, que supone la disolu-

10 Según Apel, las diferencias entre el cientificismo (que incluiría al positivismo lógico, a Popper y las diversas formas de positivismo jurídico y economicismo -neoliberal-) y el existencialismo, como filosofías dominantes en este periodo, resultan ser más bien complementarias. Apel habla incluso de una "división de trabajo": los primeros, 
ción del mundo bipolar y la globalización capitalista, se refiere al giro lingüístico-pragmático-hermenéutico en general. En otros textos, Apel habla de este contexto general como de crítica total a la razón, ${ }^{11}$ e incluye al posmodernismo en general. Aquí, en cuanto recurso interno, cuestiona de estas diferentes tradiciones filosóficas su absolutización de la tradición, comunidad e historicidad. Es en este contexto, entonces, donde podemos encontrar la reflexión de Apel sobre la tradición, la dependencia histórica y cultural del pensamiento, incluida la filosofía, respecto de la historicidad.

Si bien ha habido un desplazamiento del desafío externo desde 1945, donde la principal preocupación giraba en torno al peligro de una guerra nuclear entre las dos grandes potencias mundiales, la URSS y EEUU, aún sigue siendo imprescindible la necesidad de dar respuesta mediante una macro-ética global. Problemas como la crisis ecológica, la desigualdad o la pobreza no pueden ser abordados plena y efectivamente en el marco de las éticas tradicionales de la pequeña comu-

referidos a la esfera pública a través de una racionalidad científico-técnica, que alcanza a la teoría de juegos y de la decisión, dejan librada a sí misma la esfera privada de decisión, que queda abordada preferentemente por los existencialismos. En ambos casos, la ética normativa no tendría lugar, pues el orden social se fundamenta por una lógica técnico-científica, procedimientos autonomizados que no requieren "valoraciones", y la vida individual en última instancia remite a una "decisión" o "resolución" de la voluntad, no argumentable. Esta complementariedad ideológica no podía dar respuesta, o mejor, convertía en "sin sentido", la pregunta por la fundamentación normativa tanto de la decisión individual como del orden social. Por otro lado, la filosofía "oficial" del bloque soviético, el marxismo-leninismo, según Apel, intentó integrar las dos dicotomías del bloque capitalista: 1) la racionalidad científica libre de valores de la ciencia y la decisión valorativa irracional de la moralidad; y 2) la separación entre esfera pública de procedimientos racionales y libres de la moral y esfera privada de decisiones existenciales. Este intento de superación se logra a través de una dialéctica materialista de la historia, según la cual, la moralidad no se reduce a lo privado, sino que es un asunto de la comunidad en la realización del "objetivo de la historia": una sociedad sin clases. Aquí el partido, como vanguardia del proletariado, estaba en condiciones de exigir de cada cual el respectivo compromiso moral. Como se puede inferir, Apel es muy crítico de ambos sistemas ideológicos; sin embargo, su trabajo filosófico parte de una crítica interna de la tradición liberal-racionalista, más que de una crítica interna del marxismo-leninismo. Ver: Apel 2001 30ss.

11 Para una caracterización general de lo que Apel llama "crítica total de la razón" en el posmodernismo, ver: Scivoletto, 2012. 
nidad (micro-ética) o incluso del Estado moderno (meso-ética). Sin embargo, no sucede lo mismo con los recursos internos. La situación filosófica ha dado un giro radical. Tal giro es caracterizado por Apel como lingüístico-pragmático-hermenéutico (Apel 2001 68). En términos de la hermenéutica, esto se expresa en el descubrimiento de la preestructura del ser-en-el-mundo, lo que supone "la dependencia de nuestra comprensión del mundo de la vida de una pre-comprensión históricamente predeterminada, la cual está siempre articulada en un lenguaje; y la correspondiente dependencia de nuestra orientación moral del compartir 'valores fuertes' de una particular tradición comunitaria" (Id. 66). Tal pre-comprensión hermenéutica de nuestra comprensión del mundo, afirma Apel, converge con la idea de Wittgenstein de la imposibilidad de un lenguaje privado y de que uno solo pueda seguir una regla, esto es, que todo seguir una regla pertenece a una comunidad relativa a una forma de vida. En esta idea base se inspira, a grandes rasgos, el comunitarismo.

El comunitarismo angloamericano critica al liberalismo y las teorías deontológicas de la justicia, básicamente por su universalismo y por su desconocimiento o subestimación de la moralidad vigente (cultural, histórica, social) a partir de la cual construimos nuestros juicios morales. Ahora bien, lo que le interesa a Apel, más que un análisis detallado de las múltiples variables y diferencias (por ejemplo, entre el neoaristotelismo y neotomismo de Maclntyre, el neohegelianismo de Taylor o el comunitarismo liberal de Rorty) es una caracterización "ideal-típica" del comunitarismo y centrarse, fundamentalmente, en la idea de comunidad y en cómo, o cuáles son las posibilidades y los límites de extraer la normatividad de dicha comunidad (Apel 1994 16). Tanto la ética del discurso como la ética comunitarista parten del a priori de la comunidad; la diferencia, afirma Apel, es que la ética del discurso no parte de una comunidad particular concreta (por ejemplo, la tradición liberal norteamericana de Rorty), sino del a priori de la comunidad de comunicación. La comunidad de comunicación es real, se realiza efectivamente aquí y ahora mediante sujetos empíricos que se comunican mediante una lengua empírica, histórica, etc. Pero Apel se pregunta: 
¿Se puede comprender el a priori de la comunidad de comunicación como un haber reconocido, "ya siempre" y "en cada caso ya", presupuestos normativos de la precomprensión del mundo y del acuerdo intersubjetivo que, como "perfecto apriórico" (Heidegger), no tiene únicamente el carácter de un a priori histórico de facticidad y contingencia? (Apel 1994 17).

Lo que intenta sugerir Apel es que, en cada comunidad, en la medida en que dicha comunidad argumenta, no solo hay presupuestos fácticos contingentes relativos a una tradición, sino también presupuestos trascendentales, por lo tanto, universales y necesarios. Si la ética se fundamenta apelando al núcleo valorativo de tales comunidades, entonces, la ética no puede ser más que relativa; en cambio, si la fundamentación se extrae de aquellos presupuestos trascendentales entonces es posible una fundamentación universalista.

La perspectiva universalista remite a la tradición liberal, en la que la dimensión deontológica tiene preeminencia sobre la axiológica. Apel es parte de esa tradición, sin embargo, la fundamentación propuesta por la ética del discurso es diferente de la propuesta por la tradición liberal clásica cuestionada por el comunitarismo que, en este sentido, es heredero de las críticas románticas a la llustración. Para Apel, los motivos de la crítica comunitarista a la tradición liberal pueden ser resumidos en dos puntos: en una crítica al individualismo metodológico y en una crítica a la racionalidad instrumental estratégica (Apel, 1994 18). Más allá de la justeza de tales críticas, que Apel intenta clarificar distinguiendo los conceptos de razón y libertad en Hobbes y en Kant, lo cierto es que el comunitarismo acierta, según Apel, en la crítica a la tradición liberal, pues Kant no ha reconocido o no ha tenido en cuenta la mediación comunitaria del sentido, "sin la cual el individuo no se puede representar ninguna máxima, norma o institución" (Id. 21). En este punto Apel reconoce el gran aporte de Hegel, a través de la idea del espíritu objetivo y la eticidad sustancial. ${ }^{12}$ 
Para poder utilizar [el individuo], incluso en el sentido de la "Ilustración" kantiana, su propio entendimiento, y para poder decidir autónomamente sobre cuestiones prácticas, tiene que poder partir, ya siempre, de una comprensión previa del mundo $y$, en este sentido, también de normas convencionales y -en la moral vivida- de una "cultura de máximas" (Apel 1994 21).

No obstante, otra vez, la validez universal de las normas morales no se puede fundamentar "por recurso a la precomprensión de aquello que de hecho se hace" (Apel 1994 21). Pero tampoco se puede apelar, con Kant, al imperativo categórico "para demostrar, como aceptables por todos, normas concretas en el sentido de una legislación universal" (Id. 22). La perspectiva de la ética del discurso, como se sabe, es que la demostración de la universalidad de las normas no puede realizarse por medio de la conciencia solitaria, sino en un discurso práctico, esto es, en un diálogo argumentativo real entre los afectados o interesados, donde "las máximas" puedan alcanzar consenso. La mediación comunitaria del sentido se manifiesta, entonces, de dos maneras: mediante las máximas concretas, pertenecientes a una tradición, cuyos "valores fuertes" determinan una eticidad sustancial o normatividad concreta, y mediante el principio de universalización de formación de consenso.

A modo de conclusión de este segundo punto, se puede destacar el intento de Apel (otro punto es si tal intento logra su objetivo) de fundamentar la ética a partir de una mediación de Kant con Hegel. Esto es importante porque el propio Apel se presenta como un transformador de la ética kantiana, y esa herencia es obviamente la más visible. Sin embargo, desde los años 60, cuando Apel formulara su propuesta filosófica como una hermenéutica trascendental, la herencia kantiana viene mediada precisamente por aquella tradición. También el intento de mediación entre comunitarismo y liberalismo apunta en la misma dirección: mientras que, por un lado, Apel defiende una ética universalista que fundamenta un principio general de justicia; por otro, se 
destaca la importancia y la necesidad de la autodeterminación de lo bueno de cada comunidad concreta y de cada persona. Respecto de esto último, desde mi punto de vista, la ética del discurso es una ética más institucionalista que personalista, en el sentido que no demanda de los individuos más (ni menos) que se comporten racional-moralmente, y esto significa, fundamentalmente, no violar el principio del discurso. Pero la ética del discurso deja librada a la historia social y la autodeterminación qué proyectos de vida individuales o comunitarios son valiosos o no. El aporte de la ética del discurso consiste, como he intentado defender, en su alcance institucional, esto es, en la insistencia en la transformación de las condiciones marco para la implementación del discurso en la esfera social (administración, mercado, derecho). ${ }^{13}$

\section{La ética y el cambio social: institucionalización}

La relación de la ética con el futuro tiene que ver, desde mi punto de vista, con las posibilidades de transformación social y de realización de un determinado tipo de "ideal" o "utopía"14, aun cuando se deba distinguir rigurosamente el contenido utópico de un ideal regulativo de la utopía como forma social (empírica) de vida. En este sentido, la aplicación de la ética no puede reducirse a la resolución de conflictos o casos singulares. La función de la ética del discurso en particular no se reduce a reconstruir los criterios de legitimidad para la respuesta correcta a un conflicto de interés, a través de la fundamentación de una norma básica según la cual lo correcto ha de determinarse mediante un discurso práctico entre todos los afectados; la ética discursiva también puede aportar criterios para la transformación de las condiciones marco

\footnotetext{
13 Creo que esta institucionalización del principio del discurso también podría hacerse extensiva a los micro-ámbitos sociales, como las relaciones sociales, la familia, etc. Por ejemplo, la igualdad de género (en el caso de parejas heterosexuales) en el ámbito familiar supone la simetría discursiva de quienes componen la pareja. Si hay un "límite" a la institucionalización, esto es, dicho rápidamente, si por ejemplo la democratización de la toma de decisiones no puede ser aplicada de manera indiscriminada sin romper con la lógica interna de una determinada esfera (por ejemplo, "democratizar" el mercado) es una cuestión que no puedo abordar aquí.

14 Para la relación de la ética discursiva con la utopía, ver Apel 2004.
} 
de los discursos prácticos, esto es, para las condiciones de realización ya no formales sino materiales. Sin embargo, esta tarea es por supuesto interdisciplinaria y requiere de otros actores políticos: se requiere del aporte de las ciencias políticas, jurídicas, sociales y pedagógicas, y de los militantes, voluntarios, movimientos sociales, etc. Pero antes de avanzar en este punto quisiera analizar la forma en que Apel concibe la relación de la ética con el futuro.

En las Mercier Lectures, Apel distingue cuatro posiciones filosóficas respecto de la relación entre la ética y la dimensión temporal del futuro, a saber: la posición kantiana, la posición hegeliana, la posición historicista-relativista y la posición ético discursiva (Apel 2001 72-75). Respecto de la posición kantiana, si bien hay que distinguir, aclara Apel, el Kant sistemático del imperativo categórico (pensemos, sobre todo en la Fundamentación) y el Kant de los textos histórico-políticos, Apel interpreta esta posición como un deontologismo o ética de principios "indiferente de la situacionalidad de la moralidad dentro del proceso irreversible de la historia" (Id. 73). En otras palabras, esta posición ideal típica, que puede hacerse extensiva a toda ética de principios, se abstrae de la historia. La posición hegeliana, según Apel, representa una metafísica especulativa de la historia en la cual lo real se funde con lo necesario y, por lo tanto, el "deber ser [...] se encuentra asumido por el progreso necesario de la historia hacia un objetivo absoluto" (Id. 73). Como se podrá observar, Apel interpreta de manera convencional e incluso escolar ambas posiciones. Sin embargo, no discutiré aquí tales representaciones de la filosofía de la historia de Kant y Hegel. La tercera posición, se opone radicalmente a la metafísica de la historia, a la idea de un logos inherente a la historia que se despliega en pos de un progreso; sin embargo, según Apel, conserva el descubrimiento hegeliano de la dependencia de los juicios normativos de la historia, la tradición o eticidad concreta. Por lo que hemos visto en los puntos anteriores, esta posición respondería especialmente a la hermenéutica y también al neopragmatismo. Finalmente, la ética del discurso, fundamentada de manera pragmático-trascendental, representa la cuarta posición.

La ética del discurso, en la visión de Apel, se distancia de los dos "extremos": la abstracción total de la situación histórica de la ética 
deontológica de principios y la dependencia total a la historia de la ética hermenéutica. Respecto del primer modelo, el deontológico, la relación con la historia presenta la dificultad de que postula un punto cero de la historia o un nuevo comienzo que, en cualquier caso, es imposible. No ha existido un punto cero de la moral, así como tampoco existirá, presumiblemente, un nuevo comienzo. ${ }^{15}$ El segundo modelo, en cambio, representa el otro "extremo", entiende a la moral como completamente dependiente de la historia, y por lo tanto no hay posibilidad de una instancia crítica de fundamentación independiente de ella. La ética no puede abstraerse de la historia y refundarla, pero tampoco puede estar subordinada a ella. La diferenciación negativa, es decir, en cuanto a que no es como otras posiciones éticas que, o se abstraen de la historia o se subordinan a ella, tiene que mostrar ahora su lado propositivo. Desde la perspectiva de Apel, entonces, "la ética discursiva puede prescribir a la historia un objetivo moral, cuya realización es siempre incierta, pero un deber como tal [...] Puede prescribir, por ejemplo, la realización a largo plazo de aquellas condiciones institucionales -políticas, jurídicas y económicas- de la interacción humana que hagan posible resolver todos los conflictos de interés por medio del discurso argumentativo y no por la violencia" (Apel 2001 75).

\section{A modo de conclusión}

Las teorías éticas, o la filosofía práctica en general, pueden distinguirse por los criterios de fundamentación de sus enunciados normativos (empíricos, trascendentales, formales, materiales) o por los criterios para su aplicación. Pero cuando se habla de aplicación, generalmente, se piensa en una aplicación a casos o situaciones conflictivas actuales. Así, por ejemplo, cuando se habla de ética aplicada se piensa sobre todo en un conflicto normativo presente; sin embargo, la "aplicación" de la ética no sólo debería referirse a esta dimensión temporal, sino

15 Los escenarios postapocalípticos que ofrecen las novelas y series de ciencia ficción contemporáneas tal vez sean un síntoma de este anhelo imposible de nuestra época. 
que también deba estar articulada con el pasado y el futuro. Como he intentado defender aquí, la relación con el pasado puede ser entendida como un posicionarse frente a la moralidad vigente, la cual no es meramente las doctrinas de la época, sino su encarnación institucional. La relación con el futuro, en cambio, apunta a la transformación institucional, sea de manera reformista o revolucionaria, conforme a algún tipo de "ideal", sea empírico o meramente regulativo, inmanente o trascendente. La ética del discurso, en su versión apeliana, ocupa un lugar especial en la historia de la ética en la medida que su relación con la historia es a la vez histórica y transhistórica, es decir, la fuente de su propia normatividad y de su aplicación puede fundamentarse de manera trascendental, pero no trascendente, se encuentra también encarnada en la historia. A través del análisis de las Mercier Lectures que he llevado aquí, se puede observar cómo Apel presenta a la ética del discurso en esta doble dimensión: histórica, en la medida en que la filosofía práctica es una respuesta a la situación humana, pero trascendental en la medida que esa respuesta histórica se fundamenta en la lógica misma de la racionalidad (práctica).

El tipo de "intervención" práctica que la ética discursiva fundamenta puede ser entendida como el de la institucionalización y desinstitucionalización de condiciones marco (posibilidades reales o materiales de acceso a la esfera pública, simetría, tiempos equitativos, igualdad de trato, uso no dogmático del lenguaje, etc.). Institucionalización, en la medida en que apunta a implementar el discurso en las instituciones. Desinstitucionalización, en la medida en que cuestiona aquellas prácticas que violan el principio del discurso, que implica la igualdad (simetría) en derechos de los interlocutores y su corresponsabilidad.

\section{Bibliografía}

Anderson, J. "Competent Need-Interpretation and Discourse Ethics". Pluralism and the Pragmatic Turn. Essays in honor of Thomas McCarthy, eds. W. Rehg \& J. Bohman. MIT Press, 2001.

Apel, K.-O. La transformación de la filosofía, 2 Tomos. Madrid: Taurus, 1985. 
Apel, K.-O. Diskurs und Verantwortung. Das Problem des Übergangs Poskonventionellen Moral. Frankfurt: Suhrkamp, 1988.

Apel, K.-O. "Plurality of Good? The Problem of Affirmative Tolerance in a Multicultural Society". Ratio Juris 10(2) (1997): 199-212.

Apel, K.-O. Auseinandersetzungen in Erprobung des transzendentalpragmatischen Ansatzes, Frankfurt am Main: Suhrkamp, 1998.

Apel, K.-O. "Globalization and the Need for Universal Ethics". European Journal of Social Theory 3(2) (2000): 137-155.

Apel, K.-O. The Response of Discourse Ethics to the Moral Challenge of the Human Situation Especially Today. Leuven: Peeters, 2001.

Apel, K.-O. " ¿Es la ética de la comunidad ideal de comunicación una utopía? Acerca de la relación entre ética, utopía y crítica de la utopía. Estudios Éticos, México: Distribuciones Fontamara, (2004): 177-221.

Apel, K.-O. La globalización y una ética de la responsabilidad. Reflexiones filosóficas sobre la globalización. Buenos Aires: Prometeo Libros, 2007.Apel, K.-O., y Dussel, E. Ética del discurso y ética de la liberación. Madrid: Trotta, 2005.

Berger, P. "Arnold Gehlen and the Theory of Institutions". Social Research 32(1) (1965): 110-115.

Burckhart, H. Diskursethik - Diskursanthropologie - Diskurspädagogik. Würzburg: Königshausen \& Neumann, 1999.

Günther, K. The Sense of Appropriateness: Application Discourses in Morality and Law. New York: SUNY Press, 1993.

Honneth, A. El derecho a la libertad. Esbozo de una eticidad democrática. Buenos Aires: Katz, 2014.Maliandi, R. Cultura y conflicto. Buenos Aires: Biblos, 1984.

Maliandi, R. Ética, dilemas y convergencia. Buenos Aires: Biblos, 2006.

Michelini, D. Bien común y ética cívica. Una propuesta desde la ética del discurso. Buenos Aires, Bonum: 2008.

Niquet, M. "Die Identitäten des Menschen. Von der klassischen philosophischen Anthropologie zur Diskursanthropologie" Diskursethik und Diskursanthropologie. Aachen Vorlesungen, eds. K.-O. Apel \& M. Niquet. FreiburgMünchen: Verlag Karl Alber, 2002.

Scivoletto, G. "Repensar el humanismo y la racionalidad: aportes desde la filosofía de Karl-Otto Apel". Hermenéutica Intercultural 20-21 (2012): 105-123. 